\title{
Highlights on HIV eradication in 2013
}

\section{Antonella d'Arminio Monforte ${ }^{a}$, Valentina Svicher ${ }^{b}$, Silvia Nozzac, Adriano Lazzarin ${ }^{c}$, Giulia Marchetti ${ }^{a}$ and Carlo-Federico Perno ${ }^{b}$}

AIDS 2013, 27:000-000

\author{
Keywords: HIV-1, reservoir, HAART, HIV eradication, latent infection, \\ HIV_DNA, immune control
}

\section{Introduction}

Almost 20 years after the introduction of HAART, scientific community, doctors and patients are still struggling with the absence of effective strategies aimed at eradicating HIV infection, or at preventing it through a vaccine.

For the purpose of this opinion article, comments are grouped into virological, immunological and clinical issues.

Virological remarks for HIV cure and eradication Latently HIV-1-infected cells are rare (especially in peripheral blood), but undistinguishable from uninfected cells, thus making their clearance extremely demanding. In addition, despite several efforts, a real consensus on mechanisms by which persistent HIV-1 reservoirs are established and maintained is still lacking, thus explaining why strategies adopted so far to reverse HIV-1 latency in vivo are still incipient in terms of efficacy. So far, latency-reversing agents can induce the production of replication-competent virus from only 1 out of 1 million resting CD4+ lymphocytes. Some light on the nature of this non-induced provirus has been recently shed by Siliciano's group at last Conference on Retroviruses and Opportunistic Infections (CROI) [1]. Surprisingly, they show that around $12 \%$ of the non-induced provirus is replication-competent with intact proviral genome and long-terminal repeat (LTR) function. Notably, by investigating the sites of HIV-1 DNA integration, the authors found that this non-induced provirus preferentially integrates into introns of actively transcribed genes and lacked $\mathrm{CpG}$ methylation over LTR. The identification of this non-induced provirus, showing replication competence without evident epigenetic silencing, supports that latent reservoir can be $40-50$-folds higher than previously estimated. This may represent a clinical threat that can further increase the barrier to cure.

The scenario is further complicated by the existence of anatomical reservoirs of HIV-1 infection (Fig. 1). In this regard, the gut-associated lymphoid tissue (GALT) plays a critical role as a major viral reservoir in infected individuals. HIV-1 RNA is not detected in the gut of long-term non-progressor patients (LTNPs) (reviewed $[2,3])$, and the level of cell-associated HIV-DNA remains higher in gut than in peripheral blood mononuclear cells (PBMCs) during suppressive HAART [2], thus supporting the role of GALT in maintaining HIV-1 persistence. HIV-1-infected GALT may also play a key role in promoting microbial translocation and persistent immune activation and depletion of CD4+ lymphocytes in the blood compartment [2]. This promotes the need to identify diagnostic markers that can rapidly inform us on HIV infection status in this important anatomical reservoir.

Another topic that should be addressed is the sub-optimal penetration of antiretroviral drugs in the tissues, giving rise to a cryptic replication of HIV-1 as a further obstacle

\footnotetext{
${ }^{a}$ Department of Health Sciences, Clinic of Infectious Diseases, 'San Paolo' Hospital, University of Milan, Milan, ${ }^{b}$ Department of Experimental Medicine and Surgery, Tor Vergata University, Rome, and ${ }^{\circ}$ San Raffaele Scientific Institute, Milan, Italy.

Correspondence to Antonella d'Arminio Monforte, MD, Department of Health Sciences, Clinic of Infectious Diseases, 'San Paolo' Hospital, University of Milan, Via A. Di Rudinì, 820142 - Milan, Italy.

Tel: +3902 81843045; fax: +3902 81843054; e-mail: antonella.darminio@unimi.it
}

DOI:10.1097/01.aids.0000433241.78739.79

ISSN 0269-9370 @ 2013 Wolters Kluwer Health | Lippincott Williams \& Wilkins Copyright $@$ Lippincott Williams \& Wilkins. Unauthorized reproduction of this article is prohibited. 
In the setting of a fully suppressive HAART, residual viremia mainly derives from the release of virus from stable reservoirs

HAART initiation
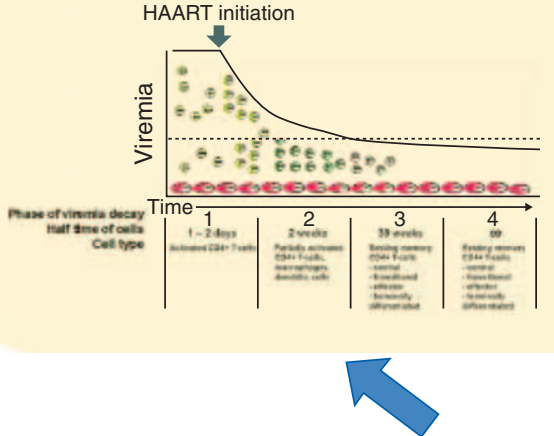

A decrease (even modest) in the level of adherence can promote residual ongoing replication. This can occur even if virological rebound is not detected.

Infected T-cells

* Viral particle

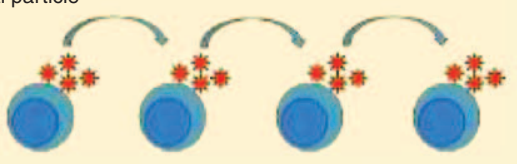

Sources of viral persistence and residual viremia despite suppressive HAART

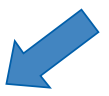

Ongoing viral replication and persistence of several forms of HIV-1 DNA in cellular reservoir and anatomical sanctuary sites.

> Some commonly used antiretroviral drugs rarely reach therapeutic concentrations in lymphatic tissues and in the gut.

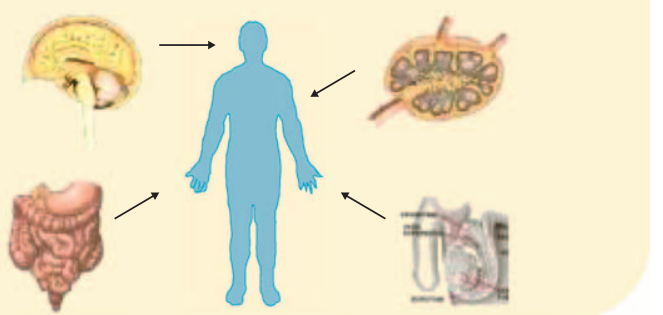

Cell-to-cell HIV transmission (occurring at the interface of two cells) is a highly efficient mode of HIV transmission particularly in tissues, and seems not to be affected by the currently available antiretroviral drugs.

Fig. 1. Sources of viral persistence despite suppressive HAART. The picture describes the main sources of HIV persistence despite suppressive HAART. Residual viraemia mainly derives from the release of viral particles from HIV-1 cellular reservoirs and cannot be reduced by intensification therapy. Even modest decrease of adherence can promote de-novo infection of cells and potential new cycles of ongoing replication, as supported by recent studies [34]. Another source of residual viraemia is represented by HIV persistence and replication in tissues where the penetration of antiretroviral drugs is suboptimal. HIV replication in tissues (particularly lymph nodes) may be also favoured by cell-to-cell transmission. Mechanisms underlying residual viraemia represent so far important obstacle for HIV functional or biological cure and highlight the need to develop diagnostic markers to measure HIV reservoir in the blood and in all anatomical compartments where the virus replicates and persists. The figure in the upper right panel has been modified from [35].

for HIV cure [4]. This is critical considering that the blood compartment contains approximately $2 \%$ of total body lymphocytes, and that some commonly used drugs (such as tenofovir and emtricitabine, just as examples) may not reach therapeutic concentrations in lymphatic tissues and in the gut where HIV replication proceeds at high rate [4]. New formulations with enhanced penetration in sanctuaries are thus needed for the success of strategies aimed at achieving HIV-1 cure.

Finally, the quest for HIV infection requires accurate, reproducible and sensitive assays to measure the burden of HIV-1 reservoirs. Cell-associated HIV-1 DNA represents the best candidate to assess such parameter. This is the right moment to set up studies aimed at validating the assays for its quantification for routine diagnostic.

\section{Immunological remarks for HIV cure and eradication}

\section{Cellular HIV reservoir}

Essential premise to eradication strategies is a thorough identification/understanding of HIV viral reservoirs in vivo. Data on both $\mathrm{HIV} /$ simian immune deficiency virus (SIV)-infected humans and animals have accumulated, detailing cell (sub)populations and life span, and tissues that can serve as viral reservoir.

Whereas memory CD4+ T-cells are the major target of HIV, HIV-infected CD4+ T-cells that escape cytotoxic T-lymphocyte (CTL) response represent the pool of lately infected resting memory CD4+ [5]. In successfully treated patients, Chomont et al. [6] described two major memory CD4+ reservoirs: central memory (TCM) and 
transitional memory (TTM), both with long halflives, whereas naïve $(\mathrm{TN})$ and terminally differentiated CD4+ (TTD) marginally contribute to the pool of cells harbouring HIV proviral DNA .

In the primate model, higher proportions of infected CD4+ TCM have been consistently shown in pathogenetic (rhesus macaques) versus non-pathogenetic (sooty mangabeys) SIV infections, whereas no differences are shown in SIV-infected CD4+ effector memory (TEM) cells; this suggests that CD4+ TCM of sooty mangabeys are relatively resistant to SIV infection, possibly reflecting the reduced CCR 5 transcription/ expression $[7,8]$. Given the central role of CD4+ TCM in long-term memory and long-lived HIV reservoir, these findings indicate that sparing CM from SIV infection is a central pathway of non-progressive infection.

Most recently, a relevant long-term reservoir for HIV in the course of HAART has been identified in T-memory stem cells (Tscm) [9], a newly identified memory T-cell subset endowed with self-renewal, homeostatic proliferation and high proliferative potentials [10]. Indeed, in HAART-treated patients, Buzon et al. [9] demonstrated higher HIV-DNA per-cell levels in Tscm versus other memory compartments, with slower decay, and identical HIV sequences after $4-8$ years of HAART, to strongly suggest heightened contribution of Tscm to total HIV reservoir and long-term persistence.

It has been extensively proven that not all anatomical sites are equally targeted by the virus. The memory lymph node-residing CD4+ T-follicular helper cell (Thf) subpopulation has been recently described to be enriched of SIV and HIV-infected cells, making them a relevant viral reservoir endowed with the highest potential for virus replication and production $[7,11]$.

Massive depletion and infection of gut-residing CCR 5+ CD4+ T-cells have been long described in SIV/HIV [12]. Furthermore, gut CD4+ infection frequencies do not appear to decrease on HAART [13]. Data with mega-HAART or raltegravir intensification, despite being controversial [14], seem to suggest that HIV reservoir in gut $\mathrm{CD} 4+$ is larger and more stable than peripheral blood.

Quite debated is the point whether intestinal macrophages constitute a reservoir; data on their permissiveness to infection are well established and confirmed in the literature, yet recent data question about their pathogenetic role $[15,16]$. Brenchley et al. [18] show no preferential depletion of CCR5+ CD4+ T-cells in the bronchoalveolar lavage (BAL) of HIV-infected individuals, with consistently lower infected memory CD4+ T-cells in BAL than peripheral blood upon HAART initiation [17]. Very low viral DNA has been shown within alveolar macrophage, to suggest low susceptibility to HIV due to reduced CCR 5 expression and not a substantial HIV reservoir [18]. On the contrary, the role of macrophages as HIV-1 cellular reservoir cannot be excluded on the basis of these results. Indeed, macrophages are resistant to HIV-1 cytopathic effect, and can persistently produce viral particles (even if at low rate due to their resting status) for prolonged period of time, thus being a persistent source of viral particles in different body compartments [19].

\section{Immune control of HIV reservoir}

Immune control over HIV reservoir can be visualized as a two-step process that requires viral transcription from latent reservoirs followed by elimination of virusproducing cells by the host innate and adaptive response, as excellently reviewed in [20]. Pro-inflammatory innate defences (e.g. type 1 interferons, chemokines), on one hand, promote the establishment of cellular HIV reservoir; whereas, on the other hand, they support ongoing immune activation, in turn favouring HIV transcription. In terms of adaptive immunity, the clonogenic and proliferating profile of different CD4+ T-cell subtypes, as well as the interconnecting effects of signals that govern CD4+ homeostasis such as IL-7, altogether dictate the exposure of HIV antigen-expressing cells to cytotoxic CD8+ clones, thus regulating the spread and composition of HIV reservoir. Under this perspective, much can be learnt by elite controllers and LTNP-natural models of functional cure. Nonprogressors might have diminished blood cellular reservoirs, as suggested by low proviral DNA in peripheral blood in elite controllers versus patients on HAART, and lower gut HIV-DNA in LTNP versus progressors; conversely, reservoir in secondary lymphoid tissues is uncertain. Limited infection of resting CD4+ TCM has been proven in human leukocyte antigen (HLA)-B27 or B57 LTNP; most interestingly, strong Gag-specific CD8+ T-cells negatively correlated with HIV reservoirs in CD4+ TCM cells [21]. Altogether, these findings support a model whereby HLA-B27 or B57-restricted CD8+ response in LTNPs and elite controllers is a crucial patrol pathway toward circulating and mucosal HIV reservoirs [20].

\section{Clinical remarks for HIV cure and eradication: may a cure for HIV infection be achievable?}

Biological cure implies the complete eradication of each single HIV-1-infected cell. There is a well known single report of a German patient who controls his HIV infection after transplant with stem cells from a donor with $\Delta 32-$ CCR 5 mutation [22]. A reproducible model of this patient could be the suppression in CCR 5 expression in HIV-1 target cells by zinc finger nuclease (ZFN)mediated gene disruption; despite intriguing data that show effective suppression in replication of CCR 5 -tropic strains of HIV-1 in animal models by CCR 5 gene knockout [23], clinical data are still poor. 
Functional cure is more feasible and aims to achieve an inactive carrier state, without viral replication and disease progression in the absence of therapy. Strategies to minimize HIV reservoir are strongly needed. Time to HAART initiation is a key factor, as early HAART blocks the increase in HIV reservoirs defined as HIV-DNA [24]. Initiating ART during primary infection (PHI) represents a major opportunity to achieve optimal immune reconstitution; in this setting, long-term HIV-1 control after treatment interruption is associated to low HIVDNA level [24,25]. A comparative analysis of cellassociated HIV-DNA from different Agence National de Recherce sur le SIDS et les hepatitesvirale (ANRS) Cohorts demonstrated reduced HIV reservoir in post-treatment controllers $(3-5$ years HAART from primary infection, long-term HIV-RNA <500copies/ml), with no changes, however, in cell type distribution [14].

Potential strategies for eradicating HIV have two potential targets, one virological and the other immunological, that can be affected by antiviral and purging/immunomodulatory agents, respectively. Compounds driven to a virological target activate transcription of quiescent HIV through different mechanisms, making it susceptible to HAART. Histone deacetylase is involved in maintaining HIV latency; its inhibitors (Histone deacetylase inhibitors, HDACis) are candidates as anti-latency drugs [26]. Vorinostat was then administered to 20 patients on stable ART, but failed to decrease PBMCs and gut HIV-DNA, suggesting that vorinostat alone is not likely to reduce lately infected cells [27].

Role of aggressive HAART regimen including raltegravir and maraviroc on HIV reservoir is controversial, because various patients may differently respond to particularly aggressive regimens, depending on a number of factors, including viraemia, HIV-DNA levels, immunoactivation status and so on. Recent data showed that double intensification with raltegravir and maraviroc is not able to decrease total HIV-DNA reservoir in peripheral blood cells [28]. This suggests that intensification does not offer added value in terms of purging HIV reservoirs; yet a recent study, led in a macaque model, tested the efficacy of a highly intensified ART (consisting of emtricitabine, tenofovir, raltegravir, ritonavir-boosted darunavir and maraviroc) in SIV-infected rhesus macaques in the pre-AIDS stage of the disease [29]. At the end of the follow-up, all animals stably displayed viral loads below 3 RNA copies/ml and HIV-DNA below 2 copies/ $510^{5}$ cells in PBMCs and rectal biopsies of all animals, and in lymph node biopsies from the majority of the study participants. In conclusion, additional studies are needed before providing a final answer regarding the role of therapy intensification for this purpose.

HIV persistence also contributes to inflammation, so immunomodulatory drugs may accelerate the decay of viral reservoir: increased immune activation may favour viral persistence, thus supporting the need of innovative interventions aimed at decreasing persistent immune activation [30]. Therapeutic options in development include chemokine receptor inhibitors, anti-fibrotic, anti-ageing, anti-inflammatory drugs and enhancer of HIV-specific immunity. Immune activation therapy could be based on cytokines such as IL-2, interferon (IFN)alfa- $2 \mathrm{~b}$ and IL-7 that have a role in maintenance of T-cell homeostasis; in particular, IL-7-activate expression of latent HIV purging quiescent HIV reservoir [31]; in Therapeutic Intensification Plus Immunomodulation in HIV-Infected Patients (ERAMUNE 01), the use of IL-7 with ART intensification was unable to decrease the HIV-DNA reservoir: the expected effect on reactivation of HIV in latently infected cells is under investigation [28]. Few data are published about the possibility to reverse immune exhaustion using antibodies against programmed cell death protein 1 (PD-1) or its ligand. The interaction between PD-1 with its ligand (PD-1L) has been shown to inhibit activation, expansion and acquisition of effector functions of HIV-specific T-cells, whereas PD-1 expression has been shown to correlate with the size of HIV-1 cellular reservoir [30]. A recent 'proof-of-concept' study in a humanized mouse model of HIV infection showed that blocking PD-L1 led to significant reductions in HIV viral load and preservation of $\mathrm{CD}^{+}{ }^{+}$T-cell counts without apparent adverse effects [32]. Currently, the AIDS Clinical Trials Group (ACTG) has in development two research protocols aimed at reversing PD-1 in virologically suppressed patients by using anti-PD1 antibody (MK-3475) and the anti-PD-L1 antibody (BMS-936559). The results of these clinical trials will shed light on the possibility to take into account immunotherapeutic interventions as an approach towards the functional cure of HIV infection.

Clinical strategies for eradication are currently objects of phase I/II clinical trials summarized in Table 1 , that are testing the efficaciousness and safety of these strategies. Few data are available about viral control after ART interruption in gene therapy and intensification strategy, and they are concordant about a partial control of viral replication, but without achieving a complete undetectability of HIV [33].

In conclusion, main viro-immunological obstacles to HIV cure despite virological-suppressive HAART include size and location of non-inducible provirus, anatomic niches enriched with HIV content with suboptimal drug penetration, preferential infection of long-lived resting memory CD4+ T-cells, in all resulting in escape from the host immune defense.

Several challenges to HIV cure have emerged: basic research on mechanisms underlying HIV latency to be rapidly translated into clinics; improving diagnostics and definition, validation and standardization of assays to measure HIV reservoirs as well as eradication markers 
Table 1. Clinical trials evaluating HIV-1 eradication.

\begin{tabular}{|c|c|c|c|c|}
\hline Target & Drug & Phase & Comments $^{\mathrm{a}}$ & Clinical trial \\
\hline $\begin{array}{l}\text { Zinc finger nuclease (ZFN)- } \\
\text { mediated gene disruption }\end{array}$ & Gene therapy & I & $\begin{array}{l}\text { In six patients the altered CD4+ cells } \\
\text { engrafted and proliferated. After } 90 \text { days, } \\
\text { up to } 7 \% \text { of peripheral blood CD } 4+\text { cells } \\
\text { showed the CCR } 5 \text { deletion }\end{array}$ & NTC01044654 \\
\hline \multirow[t]{3}{*}{ Viral transcription induction } & HDACis ; Vorinostat & $\mathrm{I} / \mathrm{II}$ & $\begin{array}{l}\text { Vorinostat in } 20 \text { patients was not } \\
\text { associated with any change in } \\
\text { HIV DNA after } 14 \text { days }\end{array}$ & NTC01319383 \\
\hline & Disulfiram & II & $\begin{array}{l}\text { In-vitro reactivation latent HIV-1 } \\
\text { expression in a primary cell } \\
\text { model of virus latency }\end{array}$ & NCT01286259 \\
\hline & Valproic acid & II & $\begin{array}{l}\text { Reduction in resting cell infection } \\
\text { seen in } 3 \text { of } 4 \text { study participants, } \\
\text { but several follow-up studies fail } \\
\text { to demonstrate sustainable effect }\end{array}$ & NCT00614458 \\
\hline \multirow[t]{2}{*}{ Drug intensification } & Raltegravir & II & $\begin{array}{l}\text { In ERAMUNE } 01 \text { no decrease of } \\
\text { HIV-DNA reservoir }\end{array}$ & NCT00515827 \\
\hline & Maraviroc & II & $\begin{array}{l}\text { In ERAMUNE } 01 \text { no decrease of } \\
\text { HIV-DNA reservoir }\end{array}$ & NCT00976404 \\
\hline \multirow[t]{2}{*}{ Immunomodulation } & Interleukin-7 & II & $\begin{array}{l}\text { In ERAMUNE } 01 \text { no decrease of } \\
\text { HIV-DNA reservoir; adverse } \\
\text { events due to antibodies }\end{array}$ & NCT01019551 \\
\hline & Sirolimus & I & $\begin{array}{l}\text { In HIV patients who have received } \\
\text { kidney transplants, sirolimus has } \\
\text { been prescribed to provide } \\
\text { immunosuppression. It enhances } \\
\text { the viral effects of HAART on HIV. } \\
\text { A major issue is the interaction } \\
\text { with HAART }\end{array}$ & ACTG5301 \\
\hline \multirow[t]{2}{*}{ Vaccination } & HIV-rAd5 vaccine & II & No data & NCT00976404 \\
\hline & HIV-pox-based vaccine & II & No data & NCT00107549 \\
\hline
\end{tabular}

${ }^{\text {a}}$ Preliminary results: preliminary data about efficacy and safety.

${ }^{\mathrm{b}}$ Histone deacetylase inhibitor.

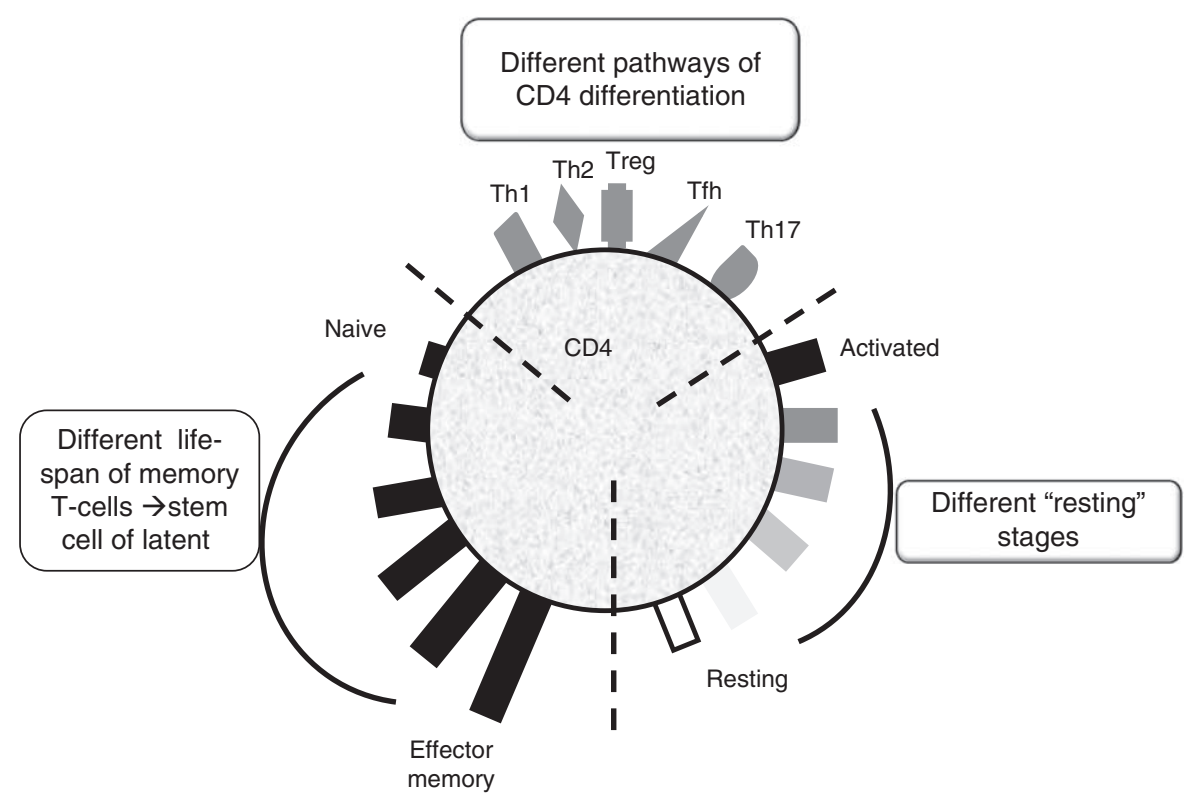

Fig. 2. Immunology of CD4+ T-cell reservoir of HIV. From an immunological standpoint, functional cure is hampered by the complex immunology of persistent reservoir within CD4+ T-cells. Different pathways of CD4+ T-cell differentiation have been proven, and their relative contribution to the establishment and persistence of the reservoirs of latently infected cells remains largely unknown. Different 'resting' stages are hypotizable within CD4+ T-cells, which could lead to different stability of the HIV reservoir. We still miss the identification of HIV reservoir 'stem cell', being endowed with higher proliferative potential, in-vivo lifespan and homeostatic impact versus the effector memory axis. (The picture was kindly provided and modified from the lecture by Dr Guido Silvestri during the Workshop on 'HIV cure and eradication: a feasible option?' - Milan, 24-25 January 2013.) 
in blood and tissues; improving drug penetration in all tissue reservoirs; thorough understanding of the complexity of the CD4+ T-cell reservoir with detailed definition of memory, activated, quiescent phenotypes and identification of 'stem cells' of HIV reservoir (Fig. 2). The ultimate challenge is the identification of 'right' patients that could achieve, without ART, stable control of HIV replication, and reversal of virus-related damage (eradication?), by combining virological and immunological strategies.

\section{Acknowledgements}

We are grateful to all the speakers of the Workshop on 'HIV cure and Eradication: a feasible option?' (Milan, 24-25 January 2013).

A. Antinori (Rome, Italy); B. Autran (Paris, France); M. Biasin (Milan, Italy); S. Bonora (Turin, Italy); J. Brenchley (Bethesda, USA); F. Ceccherini-Silberstein (Rome, Italy); P. Cinque (Milan, Italy); A. Cossarizza (Modena, Italy); S. Deeks (San Francisco, USA); G. Hutter (Mannheim, Germany); C. Katlama (Paris, France); M.M. Lederman (Cleveland, USA); J. Martinez-Picado (Barcelona, Spain); M. Puoti (Milan, Italy); C. Rouzioux (Paris, France); A. Savarino (Rome, Italy); R.-P. Sékaly (Port St Lucie, USA); G. Silvestri (Atlanta, USA); G. Tambussi (Milan, Italy).

\section{Conflicts of interest}

There are no conflicts of interest. .

\section{References}

1. Ho Y-C, Shan L, Wang J, Hosmane N, Blankson J, Siliciano R. Characterization of non-induced HIV-1 proviruses dampens the hope for HIV-1 eradication. In 20th Conference on Retroviruses and Opportunistic Infections. Atlanta, Georgia, USA; 2013.

2. Marchetti G, Tincati C, Silvestri G. Microbial translocation in the pathogenesis of HIV infection and AIDS. Clin Microbiol Rev 2013; 26:2-18.

3. Sankaran S, Guadalupe M, Reay E, George MD, Flamm J, Prindiville $\mathrm{T}$, et al. Gut mucosal $\mathrm{T}$ cell responses and gene expression correlate with protection against disease in longterm HIV-1-infected nonprogressors. Proc Natl Acad Sci U S A 2005; 102:9860-9865.

4. Cohen J. HIV/AIDS research. Tissue says blood is misleading, confusing HIV cure efforts. Science 2011; 334:1614.

5. Finzi D, Blankson J, Siliciano JD, Margolick JB, Chadwick K, Pierson $\mathrm{T}$, et al. Latent infection of CD4+ $\mathrm{T}$ cells provides a mechanism for lifelong persistence of HIV-1, even in patients on effective combination therapy. Nat Med 1999; 5:512-517.

6. Chomont N, El-Far M, Ancuta P, Trautmann L, Procopio FA, Yassine-Diab B, et al. HIV reservoir size and persistence are driven by $\mathbf{T}$ cell survival and homeostatic proliferation. Nat Med 2009; 15:893-900.

7. Brenchley JM, Vinton C, Tabb B, Hao XP, Connick E, Paiardini $M$, et al. Differential infection patterns of CD4+ T cells and lymphoid tissue viral burden distinguish progressive and nonprogressive lentiviral infections. Blood 2012; 120:4172 4181.
8. Paiardini M, Cervasi B, Reyes-Aviles E, Micci L, Ortiz AM, Chahroudi $A$, et al. Low levels of SIV infection in sooty mangabey central memory $\mathrm{CD}^{4+} \mathrm{T}$ cells are associated with limited CCR5 expression. Nat Med 2011; 17:830-836.

9. Buzon M, Sun H, Li C, Martin-Gayo E, Seiss K, Shaw A, et al. T memory stem cells are a long-term reservoir for HIV1. In 20th Conference on Retroviruses and Opportunistic Infections. Atlanta, Georgia, USA; 2013.

10. Gattinoni L, Lugli E, Ji Y, Pos Z, Paulos CM, Quigley MF, et al. A human memory $T$ cell subset with stem cell-like properties. Nat Med 2011; 17:1290-1297.

11. Perreau M, Savoye AL, De Crignis E, Corpataux JM, Cubas R, Haddad EK, et al. Follicular helper $\mathbf{T}$ cells serve as the major CD4 T cell compartment for HIV-1 infection, replication, and production. J Exp Med 2013; 210:143-156.

12. Brenchley JM, Price DA, Douek DC. HIV disease: fallout from a mucosal catastrophe? Nat Immunol 2006; 7:235-239.

13. Chun TW, Nickle DC, Justement JS, Meyers JH, Roby G, Hallahan CW, et al. Persistence of HIV in gut-associated lymphoid tissue despite long-term antiretroviral therapy. J Infect Dis 2008; 197:714-720.

14. Lewin SR, Rouzioux C. HIV cure and eradication: how will we get from the laboratory to effective clinical trials? AIDS 2011; 25:885-897.

15. Shen R, Richter HE, Clements RH, Novak L, Huff K, Bimczok D, et al. Macrophages in vaginal but not intestinal mucosa are monocyte-like and permissive to human immunodeficiency virus type 1 infection. / Virol 2009; 83:3258-3267.

16. Moore AC, Bixler SL, Lewis MG, Verthelyi D, Mattapallil JJ. Mucosal and peripheral Lin- HLA-DR+ CD11c/123- CD13+ CD14- mononuclear cells are preferentially infected during acute simian immunodeficiency virus infection. / Virol 2012; 86:1069-1078.

17. Knox KS, Vinton C, Hage CA, Kohli LM, Twigg HL, Klatt NR, et al. Reconstitution of CD4 T cells in bronchoalveolar lavage fluid after initiation of highly active antiretroviral therapy. J Virol 2010; 84:9010-9018.

18. Brenchley JM, Knox KS, Asher Al, Price DA, Kohli LM, Gostick $E$, et al. High frequencies of polyfunctional HIVspecific T cells are associated with preservation of mucosal CD4 T cells in bronchoalveolar lavage. Mucosal Immunol 2008; 1:49-58.

19. Garaci E, Aquaro S, Lapenta C, Amendola A, Spada M, Covaceuszach $\mathrm{S}$, et al. Antinerve growth factor $\mathbf{A b}$ abrogates macrophage-mediated HIV-1 infection and depletion of CD4+ T lymphocytes in hu-SCID mice. Proc Natl Acad Sci U S A 2003; 100:8927-8932.

20. Autran B, Descours B, Bacchus C. Immune control of HIV-1 reservoirs. Curr Opin HIV AIDS 2013; 8:204-210.

21. Autran B, Descours B, Avettand-Fenoel V, Rouzioux C. Elite controllers as a model of functional cure. Curr Opin HIV AIDS 2011; 6:181-187.

22. Hütter G, Nowak D, Mossner M, Ganepola $S$, Müssig $A$, Allers K, et al. Long-term control of HIV by CCR5 Delta32/ Delta32 stem-cell transplantation. $N$ Engl J Med 2009; 360: 692-698.

23. Cannon $\mathrm{P}$, June $\mathrm{C}$. Chemokine receptor $\mathbf{5}$ knockout strategies. Curr Opin HIV AIDS 2011; 6:74-79.

24. Hocqueloux L, Avettand-Fènoël $V$, Jacquot $S$, Prazuck $T$, Legac E, Mélard A, et al. Long-term antiretroviral therapy initiated during primary HIV-1 infection is key to achieving both low HIV reservoirs and normal $\mathbf{T}$ cell counts. I Antimicrob Chemother 2013.

25. Sáez-Cirión A, Bacchus C, Hocqueloux L, Avettand-Fenoel V, Girault I, Lecuroux C, et al. Post-treatment HIV-1 controllers with a long-term virological remission after the interruption of early initiated antiretroviral therapy ANRS VISCONTI Study. PLoS Pathog 2013; 9:e1003211.

26. Rasmussen TA, Schmeltz Søgaard O, Brinkmann C, Wightman F, Lewin S, Melchjorsen J, et al. Comparison of HDAC inhibitors in clinical development: effect on HIV production in latently infected cells and T-cell activation. Hum Vaccin Immunother 2013:993.

27. Elliot J, Solomon A, Wightman F, Smith M, Palmer S, Prince M et al. The safety and effect of multiple doses of vorinostat on HIV transcription in HIV+ patients receiving CART. In 20th Conference on Retroviruses and Opportunistic Infections. Atlanta, Georgia, USA; 2013. 
28. Katlama C, Lambert S, Assoumou L, Papagno L, Lecardonnel F, Tambussi G, et al. Impact of interleukin-7 and raltegravir + maraviroc intensification on total HIV DNA reservoir: results from ERAMUNE 01. In 20th Conference on Retroviruses and Opportunistic Infections. Atlanta, Georgia, USA; 2013.

29. Shytaj IL, Norelli S, Chirullo B, Della Corte A, Collins M, YalleyOgunro J, et al. A highly intensified ART regimen induces longterm viral suppression and restriction of the viral reservoir in a simian AIDS model. PLoS Pathog 2012; 8:e1002774.

30. Hatano $\mathrm{H}$. Immune activation and HIV persistence: considerations for novel therapeutic interventions. Curr Opin HIV AIDS 2013; 8:211-216.

31. Sereti I, Dunham RM, Spritzler J, Aga E, Proschan MA, Medvik $\mathrm{K}$, et al. IL-7 administration drives $\mathrm{T}$ cell-cycle entry and expansion in HIV-1 infection. Blood 2009; 113:6304-6314.
32. Palmer BE, Neff CP, Lecureux J, Ehler A, Dsouza M, RemlingMulder $L$, et al. In vivo blockade of the PD-1 receptor suppresses HIV-1 viral loads and improves CD4+ T cell levels in humanized mice. I Immunol 2013; 190:211-219.

33. Lewis MG, DaFonseca S, Chomont N, Palamara AT, Tardugno $\mathrm{M}$, Mai $\mathrm{A}$, et al. Gold drug auranofin restricts the viral reservoir in the monkey AIDS model and induces containment of viral load following ART suspension. AIDS 2011; 25:1347-1356.

34. Pasternak AO, de Bruin M, Jurriaans $S$, Bakker $M$, Berkhout $B$, Prins JM, et al. Modest nonadherence to antiretroviral therapy promotes residual HIV-1 replication in the absence of virological rebound in plasma. J Infect Dis 2012; 206:1443-1452.

35. Palmer S, Josefsson L, Coffin JM. HIV reservoirs and the possibility of a cure for HIV infection. I Intern Med 2011; 270:550-560. 